Economía Agraria y Recursos Naturales. ISSN: 1578-0732. Vol. 8, 1. (2008), pp. 57-82

\title{
Asimetrías en la transmisión de precios en el sector del tomate en España
}

\author{
Monia Ben Kaabia ${ }^{\mathrm{a}}$ y José María Gil Roig ${ }^{\mathrm{b}}$
}

\begin{abstract}
RESUMEN: El objetivo de este trabajo consiste en analizar el proceso de transmisión de precios a lo largo de la cadena comercial en el sector del tomate en España. Para ello se han considerado el precio percibido por el productor y el pagado por el consumidor. El enfoque metodológico adoptado se basa en la estimación de un Vector de Corrección del Error con umbrales. Los resultados indican que, a largo plazo, los dos precios son homogéneos. Sin embargo, en el corto plazo las reacciones de precios, tanto en velocidad como en magnitud, son asimétricas. Es más, los resultados indican que los detallistas se benefician (en el sentido de que son capaces de aumentar el margen comercial) de cualquier shock, ya sea positivo o negativo, que afecte tanto a las condiciones de oferta como de demanda. Esta capacidad de actuación sobre los márgenes comerciales es uno de los principales determinantes de los procesos inflacionistas en este sector.
\end{abstract}

PALABRAS CLAVE: Asimetrías, cointegración por umbrales, precios, tomate.

Clasificación JEL: C32, Q13.

\section{Price transmission asymmetries in the Spanish tomato sector}

SUMMARY: This paper aims to investigate the non-linear adjustments between farm and retail prices in the tomato sector in Spain. The methodology used is based on the multivariate approach to specify and estimate a Threshold Autoregressive Model. The results indicate that, in the long run, price transmission is perfect. In the short-run, price adjustments between the farm and the retail levels are asymmetric. Retailers always benefit (in terms of increasing marketing margins) from positive and negative shocks affecting supply or demand conditions. Moreover, marketing margins have been found to be main determinants of inflation in the Spanish tomato sector.

KEYWORDS: Asymmetries, threshold cointegration, prices, tomato.

JEL classification: C32, Q13.

\footnotetext{
a Departamento de Análisis Económico. Universidad de Zaragoza

b CREDA-UPC-IRTA
}

Dirigir correspondencia a: José María Gil. E-mail: chema.gil@upc.edu

Recibido en octubre de 2006. Aceptado en marzo de 2008. 


\section{Introducción}

El análisis de la transmisión de precios a lo largo de la cadena comercial es un tema que ha despertado un gran interés entre los economistas agrarios. Los precios son, quizás, el principal mecanismo que permite relacionar los diferentes eslabones de la cadena comercial, por lo que el análisis de la transmisión de precios se ha utilizado como medio para evaluar el funcionamiento general de los mercados. Este interés ha aumentado en los últimos años como consecuencia de los cada vez más complejos procesos de concentración, tanto horizontal como vertical, que han tenido lugar en los mercados agroalimentarios. En este contexto, los estudios sobre transmisión de precios pueden considerarse como una respuesta de los economistas agrarios ante la creciente preocupación social y pública sobre las consecuencias de dichos procesos de concentración.

Uno de los aspectos a los que se ha prestado más atención en los últimos años es a la posible existencia de asimetrías en los mecanismos de transmisión de precios a lo largo de la cadena alimentaria. El concepto de «asimetría» se asocia tanto a la magnitud como a la velocidad de respuesta de los diferentes precios. Aunque la existencia de asimetrías en la transmisión de precios no tiene por qué asociarse a mercados ineficientes, lo que sí parece claro es que las respuestas rápidas y simétricas de los precios antes shocks inesperados, tanto de oferta como de demanda, suelen considerarse como representativas de mercados eficientes.

Dado que la teoría tradicional de los mercados agrarios asume la existencia de ajustes simétricos en precios, el conocimiento que se ha ido obteniendo sobre la posible existencia de asimetrías en la transmisión de precios procede de la acumulación de análisis empíricos. A partir de dichos trabajos, son numerosas las posibles causas que los investigadores han identificado para explicar la existencia de relaciones de precios asimétricas [Meyer y von Cramon-Taubadel (2004)]. Entre las más comunes, podemos destacar: el poder de mercado del sector detallista [Bailey y Brorsen (1989); Griffith y Piggott (1994); entre otros $]^{1}$; los costes de transacción a nivel detallista [Chavas y Mehta (2002)]; el grado de sustitutibilidad de inputs en el proceso productivo [Bettendorf y Verboven (2000)]; el nivel de inventarios tanto a nivel productor como detallista [Reagan y Witzman (1982)]; y la intervención pública [Kinnucan and Forker (1987)].

En cualquier caso, la literatura empírica no ofrece resultados concluyentes en una determinada dirección. Las conclusiones obtenidas difieren de un trabajo a otro dependiendo del producto analizado, del país de estudio, de la frecuencia de los datos,

\footnotetext{
1 A pesar de que en la mayor parte de los trabajos, la existencia de asimetrías en la transmisión de precios se ha relacionado con comportamientos no competitivos, McCorriston et al. (2001) demostraron que esto no tenía por qué ser cierto siempre y que, en todo caso, había que tener en cuenta la relación entre poder de mercado y la existencia de economías de escala. Si la función de costes del detallista se caracteriza por la existencia de rendimientos de escala decrecientes, el efecto del poder de mercado se refuerza, provocando ajustes asimétricos. Sin embargo, en el caso de que la función de costes se caracterice por la existencia de rendimientos de escala crecientes, el efecto del poder de mercado se compensa, pudiendo aparecer ajustes simétricos.
} 
del período muestral y, sobre todo, del método de análisis empleado. En este sentido, y teniendo en cuenta los desarrollos que habían tenido lugar en las últimas dos décadas en el ámbito del análisis de series temporales, Von Cramon-Taubadel (1998) demostró, en un artículo pionero en este campo, que el enfoque tradicionalmente utilizado hasta la fecha, basado en los trabajos de Wolffram (1971), Houck (1977) y Ward (1982), no era compatible con la posible existencia de precios no estacionarios y cointegrados, especificando un modelo en forma de corrección del error asimétrico que era capaz de recoger la posible existencia de asimetrías tanto en el corto como en el largo plazo. Sin embargo, la existencia de costes de transacción puede generar ajustes en precios no lineales, que no pueden ser recogidos por un modelo lineal de corrección del error. En este sentido, los modelos por umbrales se han convertido, en los últimos años, en el enfoque metodológico más adecuado para analizar las asimetrías en la transmisión de precios [Azzam (1999)].

El objetivo de este trabajo consiste en analizar, utilizando el enfoque de modelos por umbrales, el mecanismo de transmisión de precios, tanto a corto como a largo plazo, en el sector del tomate en España. En concreto, partiendo de una situación de equilibrio en la que se produzca un shock inesperado en cualquiera de los dos extremos de la cadena comercial (productor-consumidor), enfocaremos nuestro trabajo hacia cuatro aspectos: 1) la magnitud de la respuestas en los dos niveles de la cadena; 2) la velocidad de ajuste hacia el equilibrio tras el shock en los dos extremos; 3) la naturaleza simétrica o asimétrica de la respuesta; y 4) la dirección de la respuesta, esto es, si los ajustes son diferentes dependiendo si el shock se transmite hacia arriba o hacia abajo de la cadena comercial.

Para la consecución del mencionado objetivo, el trabajo se ha estructurado en cuatro apartados adicionales. En el apartado 2, describimos brevemente las características más relevantes del sector del tomate en España, de forma que nos permitan ayudar a explicar algunos de los resultados de la aplicación empírica. El apartado 3 recoge el enfoque metodológico adoptado en este trabajo. Los principales resultados se presentan en el apartado 4. El trabajo concluye con una serie de consideraciones finales en las que se exploran posibles líneas de investigación futuras.

\section{Algunos aspectos relevantes del sector del tomate en España}

España es el segundo país productor de tomate de la UE, detrás de Italia, con una producción cercana a los 4 millones de toneladas, en el año 2003. La producción española tiene una clara tendencia exportadora ya que alrededor del $25 \%$ de la producción (0,95 millones de toneladas) se vende fuera de nuestras fronteras. Entre 1990 y 2004, la producción ha crecido en algo más de un millón de toneladas (MAPA, 2006), principalmente debido a la introducción de innovaciones tecnológicas (producción bajo plástico) que han generado un aumento notable de los rendimientos (de 45 t/ha, en 1990, a 64 t/ha, en 2004), si bien la superficie cultivada también ha crecido ligeramente. Según datos del Ministerio de Agricultura, Pesca y Alimentación (www.mapya.es), un $60 \%$ de la producción se destina a consumo en fresco, mientras 
que el $40 \%$ restante va a la industria de transformación. En este trabajo únicamente nos vamos a referir a la producción destinada al consumo en fresco.

El sector productor de tomate en España se encuentra bastante atomizado (MAPA, 2006), con algo más de 10.000 operadores. Se trata de un sector dual. A pesar de que todavía una parte importante de los productores están poco cohesionados, un porcentaje creciente se encuentra integrado verticalmente, sobre todo aquellos con una orientación exportadora. La comercialización del tomate responde a dos estrategias diferenciadas teniendo en cuenta el mencionado carácter dual de la producción. Así, podemos diferenciar entre una cadena de valor tradicional, en la que los mayoristas en origen y destino continúan jugando un papel predominante, y una cadena de valor moderna con tres agentes principales: las asociaciones de productores, la distribución y los operadores logísticos que trabajan para los dos agentes anteriores.

Teniendo en cuenta las características estructurales del sector y la escasa incidencia de la legislación europea sobre la regulación de los mercados, podemos decir que, en términos generales, los precios en los diferentes niveles de la cadena comercial se determinan por la ley de la oferta y la demanda. El conocimiento de la magnitud y la duración de los efectos generados nos permitirán determinar cómo es el mecanismo de transmisión de precios a lo largo de la cadena comercial. El Gráfico 1 muestra la evolución de los precios al productor ${ }^{2}$ y al consumo del tomate en España, desde enero de 1986 hasta junio de 2004. Como se puede apreciar, ambos precios han experimentado un crecimiento sostenido a lo largo del período considerado, siendo mucho más evidente a partir de 1997. Asimismo, se puede comprobar la existencia de un marcado componente estacional coincidente con el final de la campaña de recolección estival. Finalmente, la simple observación de la evolución de ambos precios parece indicar un crecimiento sostenido del margen comercial.

A pesar de que el aumento del diferencial de precios podría llevar al lector a pensar en una pérdida del poder de mercado del sector productor, un análisis más pormenorizado de la situación nos lleva a matizar mucho esta conclusión que, por otra parte, ha sido muy común en la mayor parte de los trabajos de divulgación y medios de comunicación. En el Gráfico 2 se recoge, utilizando medias anuales para el período considerado, la evolución del diferencial de precios en relación con el precio percibido por el productor. Se han elaborado dos tipos de medidas relativas: la primera, considerando todas las cotizaciones mensuales; y la segunda, eliminando los meses finales de la campaña estival (agosto y septiembre), esto es, eliminando la información de los meses con una cotización más elevada. Como se puede apreciar, sobre todo en el segundo caso, a pesar de la existencia de ciclos de uno o dos años, el margen comercial se ha mantenido estable, alrededor de un 155\%, a lo largo del período de estudio. Este resultado parece indicar que los operadores han trabajado con márgenes proporcionales estables a lo largo del tiempo. Esto explicaría el aumento

${ }^{2}$ Los precios al productor y al consumidor no son directamente comparables, dado que a lo largo de la comercialización se producen mermas y pérdidas. A efectos de comparar dos bienes homogéneos, hemos aplicado un factor de conversión de 1,08 al precio del productor, siguiendo las recomendaciones del United States Department of Agriculture (USDA), recogidas en Sanjuán et al. (1996). 


\section{GRÁFICO 1}

Evolución mensual de los precios de tomate en España $(€ / \mathrm{kg})$

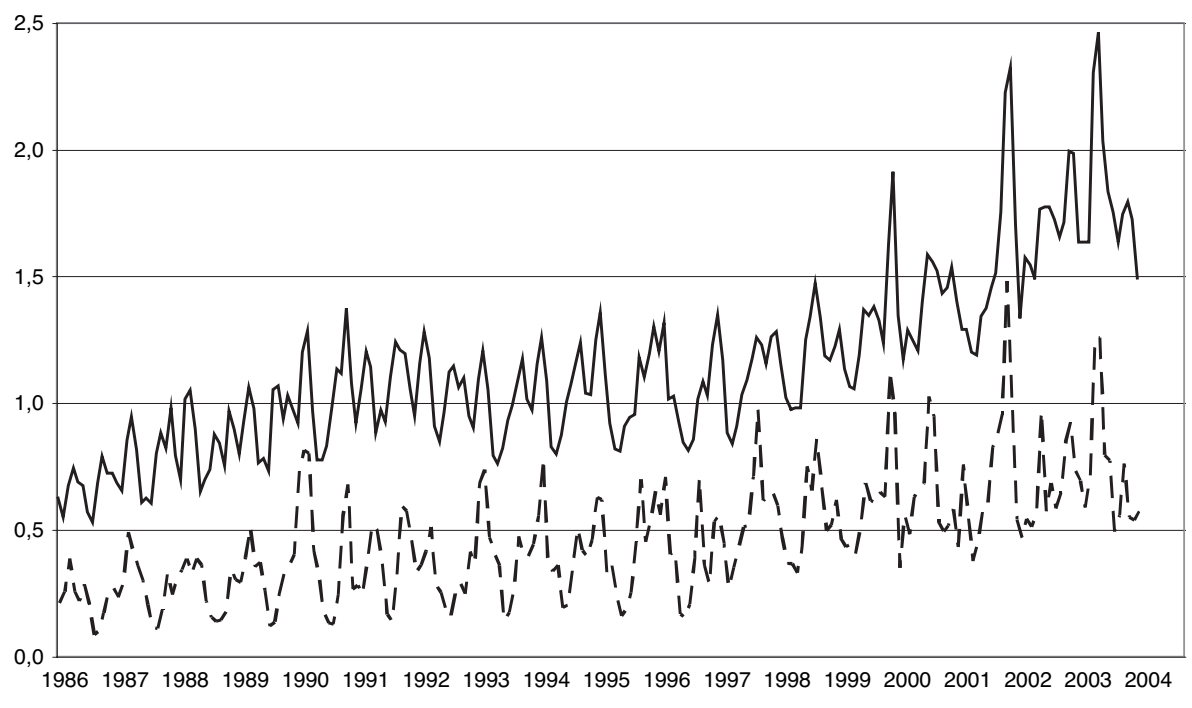

- Precio al consumo - - Precio al productor

Fuente: www.mapya.es y elaboración propia.

\section{GRÁFICO 2}

Evolución del precio al productor y del margen comercial en el sector del tomate en España (€/KG y \% sobre precio al productor)

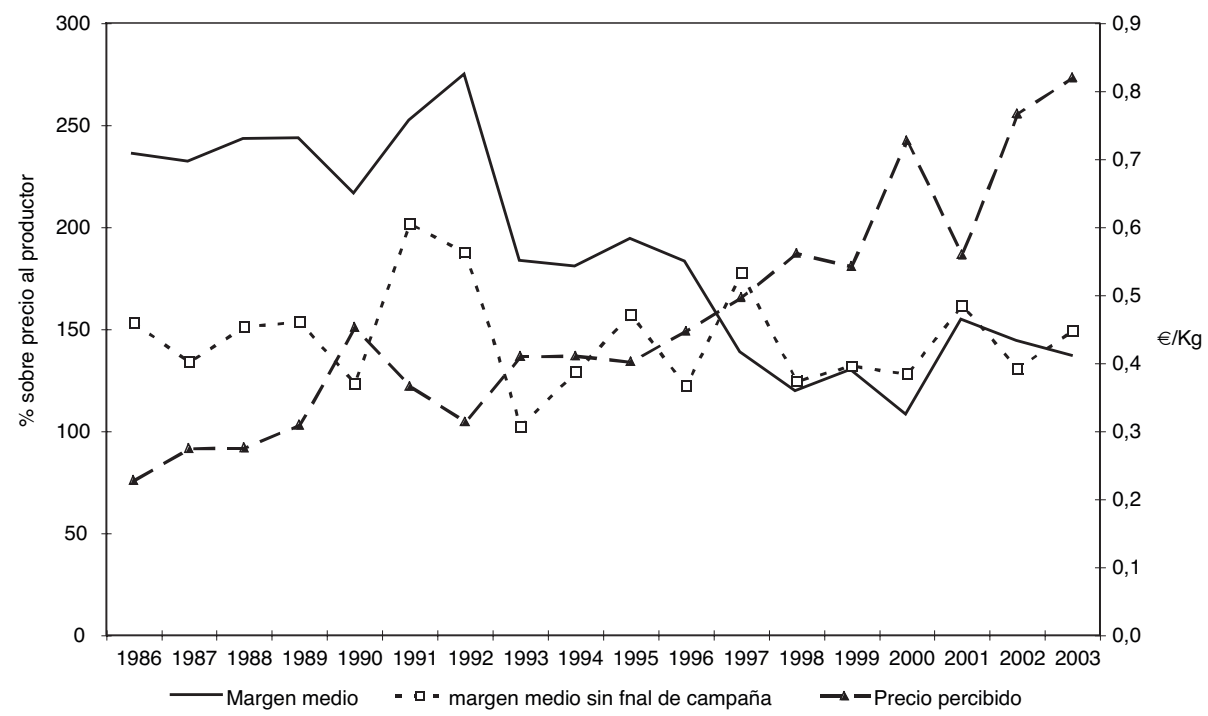

Fuente: www.mapya.es y elaboración propia. 
del diferencial de precios absoluto, tal como se indica en el Gráfico 1, conforme ha venido aumentando el precio percibido.

Para concluir este apartado descriptivo, y teniendo en cuenta el objetivo de este trabajo, a partir de las medias anuales, podemos entresacar una conclusión importante. Conforme aumentan los precios percibidos, el margen disminuye y viceversa. Por el contrario, precios a la baja significan un aumento del diferencial de precios. En el largo plazo el diferencial de precios se mantiene estable, tal como hemos indicado anteriormente. Estos resultados intuitivos son los que trataremos, precisamente, de formalizar y contrastar en los próximos apartados.

\section{Enfoque metodológico}

Tradicionalmente, el análisis de la transmisión de precios, en la que explícitamente se considera la posibilidad de existencia de ajustes asimétricos entre los diferentes precios, se ha realizado empleando la metodología propuesta por Houck (1977) y Ward (1982), a partir de un trabajo anterior de Wolffram (1971). Dicho enfoque constituye el marco adecuado para analizar el mecanismo de transmisión de precios a lo largo de la cadena comercial siempre que las series de precios sean estacionarias. Sin embargo, la mayor parte de las series económicas son no estacionarias (su varianza no es invariante en el tiempo), si bien pueden existir entre ellas relaciones estables en el largo plazo (cointegración). Granger y Lee (1989) propusieron un Mecanismo de Corrección del Error asimétrico (MCEA), en el que la velocidad del ajuste de la variable endógena (por ejemplo el precio al consumo) dependía del valor positivo o negativo de la desviación del equilibrio a largo plazo. Basándose en esta idea, Von Cramon-Taubadel (1998), Balke et al . (1998) y Frost y Bowden (1999) propusieron, bajo la hipótesis de series cointegradas, especificar el modelo de WolfframHouck-Ward como un modelo de corrección del error para contrastar la posible presencia de relaciones asimétricas entre precios a lo largo de la cadena comercial.

Una de las limitaciones más importantes que presenta este enfoque es que asume que los ajustes en precios son lineales. Sin embargo, la presencia de costes de transacción fijos puede generar reacciones no lineales; esto es, los procesos de ajuste en precios no sólo pueden diferir dependiendo del signo de la variación inicial sino que también la magnitud de la misma puede ser relevante. Por poner un ejemplo, y asumiendo que el proceso de ajuste es vía coste, no es irreal asumir que los detallistas únicamente modificarán el precio al consumo si la variación del precio percibido es realmente significativa (si supera un determinado umbral). Variaciones pequeñas, ya sean positivas o negativas pueden no dar lugar a reacción alguna debido, como hemos mencionado anteriormente, a la posible existencia de costes de transacción fijos.

Esta limitación ha dado lugar al desarrollo de propuestas alternativas capaces de compatibilizar la existencia de ajustes asimétricos y la presencia de relaciones de cointegración entre las variables. Recientemente, Chavas y Metha (2004) proponen un modelo de corrección del error ampliado que permite que la dinámica de ajuste de los precios pueda diferir según los diferentes regímenes definidos por los correspondientes umbrales. Sin embargo, en su modelo, la determinación de los umbrales se 
realiza de forma exógena. De ahí que en la actualidad hayan gozado de una mayor popularidad modelos más generales que permiten la determinación endógena de los umbrales. Entre estos, los más utilizados son: el modelo autorregresivo con regímenes cambiantes Markovianos (Markov Switching Autoregressive Model) (MS-AR) [Hansen (1997)], el modelo autorregresivo por umbrales (Threshold Autoregressive Model) (TAR) [Balke y Fomby (1997); Obstfeld y Taylor (1997); Goodwin y Piggott (2001)], y el Vector de Corrección del Error por umbrales (Threshold Vector Error Correction Model) (TVECM) [Ben-Kaabia y Gil (2007)]; Lo and Zivot (2001); Goodwin and Piggott (2001) y Hansen y Seo (2002)]. En este trabajo, la transmisión de precios en el sector del tomate en España se va a realizar a partir de la especificación y estimación de un modelo TVECM, cuya descripción presentamos en los párrafos siguientes ${ }^{3}$.

Sea $P_{t}=\left(p_{t}^{p}, p_{t}^{c}\right)^{\prime}$ un vector que recoge la evolución del precio percibido por el productor y pagado por el consumidor, ambos expresados en logaritmos, de un determinado producto. Asimismo, asumamos que $P_{t}$ es un vector de series I(1) que están cointegradas y cuyo vector de cointegración viene dado por $\beta^{\prime}=\left(1,-\beta_{2}\right)$. Bajo estas condiciones la relación entre ambos precios se puede representar mediante un Vector en forma de Modelo de Corrección del Error lineal, que viene dado por:

$$
\Delta \mathrm{P}_{\mathrm{t}}=\alpha\left[\omega_{\mathrm{t}-1}(\beta)\right]+\sum_{\mathrm{i}=1}^{\mathrm{k}-1} \Gamma_{\mathrm{i}} \Delta \mathrm{P}_{\mathrm{t}-\mathrm{i}}+\varepsilon_{\mathrm{t}}
$$

donde k indica el número de retardos, $\omega_{t}(\beta)=\beta^{\prime} P_{t-1}$ representa la relación de equilibrio a largo plazo existente entre los dos precios, evaluada en el punto genérico $\beta=\left(1,-\beta_{2}\right)^{\prime} ; \Gamma_{i}, i=1,2 \ldots$ son matrices de orden $(2 \times 2)$ de parámetros a corto plazo; $\alpha$ es una matriz de orden $(2 \times 1)$; y $\varepsilon_{t}$ es el vector de términos de error que se supone se distribuyen de forma idéntica e independiente según una distribución Gausiana, con una matriz de varianzas y covarianzas $S$ definida positiva.

Siguiendo a Lo y Zivot (2001), la especificación general de un Vector en forma de Modelo de Corrección por umbrales con tres regímenes $\left(\mathrm{TVECM}_{3}\right)^{4}$, puede escribirse como:

\footnotetext{
${ }^{3}$ Los modelos TAR y TVECM se han convertido en un procedimiento muy popular dado que son relativamente más simples de especificar, estimar e interpretar que muchos otros modelos de series temporales no lineales (Hansen, 1997). El modelo TAR se basa en una estrategia en dos etapas, similar a la propuesta de Engle y Granger (1987), en el caso del análisis de cointegración. Asimismo, asume la existencia de una relación causal entre la variable dependiente y la variable independiente. El modelo TVECM genera estimaciones más robustas que el modelo TAR (Hansen y Seo, 2002) a la vez que considera como endógenas a las dos series de precios incluidas en el modelo. Una excelente descripción de los modelos de cointegración por umbrales puede encontrarse en Gonzalo y Pitarakis (2006).

${ }^{4}$ Los estudios sobre asimetrías en la transmisión de precios han considerado, o bien la existencia de un único umbral, diferenciando dos regímenes de ajuste en precios (Balke y Fomby, 1997; Enders y Granger, 1998, Abdulai, 2002; Hansen y Seo, 2002), o bien la existencia de dos umbrales (tres regímenes de ajuste) (Obstfeld y Taylor, 1997; Goodwin y Piggott, 2001; Serra y Goodwin, 2002; entre otros).
} 


$$
\Delta \mathrm{P}_{\mathrm{t}}=\left\{\begin{array}{lll}
\alpha^{1} \omega_{\mathrm{t}-1}(\beta)+\sum_{\mathrm{i}=1}^{\mathrm{k}-1} \Gamma_{\mathrm{i}}^{1} \Delta \mathrm{P}_{\mathrm{t}-\mathrm{i}}+\varepsilon_{\mathrm{t}}^{1}, & \text { si } & \omega_{\mathrm{t}-1}(\beta)<\lambda^{1} \\
\alpha^{2} \omega_{\mathrm{t}-1}(\beta)+\sum_{\mathrm{i}=1}^{\mathrm{k}-1} \Gamma_{\mathrm{i}}^{2} \Delta \mathrm{P}_{\mathrm{t}-\mathrm{i}}+\varepsilon_{\mathrm{t}}^{2}, & \text { si } & \lambda^{1} \leq \omega_{\mathrm{t}-1}(\beta) \leq \lambda^{2} \\
\alpha^{3} \omega_{\mathrm{t}-1}(\beta)+\sum_{\mathrm{i}=1}^{\mathrm{k}-1} \Gamma_{\mathrm{i}}^{3} \Delta \mathrm{P}_{\mathrm{t}-\mathrm{i}}+\varepsilon_{\mathrm{t}}^{3}, & \text { si } & \omega_{\mathrm{t}-1}(\beta)>\lambda^{2}
\end{array}\right.
$$

donde $\omega_{t-1}(\beta)$ es la variable que se utiliza como referencia para definir los diferentes regímenes y que no es sino el residuo de la relación de equilibrio a largo plazo entre los dos precios (o, en otras palabras, la desviación respecto a dicho equilibrio), y $\lambda=\left(\lambda^{1} \lambda^{2}\right)$ es el vector que recoge los dos umbrales que permiten diferenciar entre los tres regímenes. Como puede observarse en la expresión [2], ante cualquier shock inesperado en $\omega_{t-1}(\beta)$, el ajuste que se produce en cada precio para recuperar el equilibrio a largo plazo difiere según el régimen en el que nos encontremos.

Si en [2] los umbrales $\left(\lambda^{1}\right.$ y $\left.\lambda^{2}\right)$ fueran conocidos, el modelo resultante sería lineal y, si el vector de perturbaciones cumple las hipótesis clásicas, podría estimarse mediante el método de mínimos cuadrados multivariantes. Sin embargo, normalmente dichos parámetros son desconocidos, por lo que deben estimarse conjuntamente con el resto de parámetros del modelo. A partir de los trabajos de Tsay (1998) y de Hansen (1999), Lo y Zivot (2001) proponen una estrategia para estimar un modelo como el definido en [2].

El proceso se inicia definiendo un proceso de búsqueda bi-dimensional dando valores a los dos umbrales $\left(\lambda^{1}, \lambda^{2}\right)$. El número de observaciones en cada régimen suele acotarse de tal forma que $T_{i} / T \geq \pi_{0}$ donde $T_{i}$ es el número de observaciones existentes en el régimen $i$-ésimo y $T$ es el número total de observaciones. Andrews (1993) sugiere tomar valores de $\pi_{0}$ en el intervalo $(0,05 ; 0,15)$. Los valores finalmente estimados de los umbrales son aquellos que optimizan la siguiente función:

$$
\left(\hat{\lambda}^{1}, \hat{\lambda}^{2}\right)=\arg \min _{\lambda \in\left[\lambda^{L}, \lambda^{v}\right]}\left(\Sigma_{3}\left(\lambda^{1}, \lambda^{2}\right)\right)
$$

donde $\Sigma_{3}\left(\lambda^{1}, \lambda^{2}\right)=\ln \left|\hat{\Sigma}\left(\lambda^{1}, \lambda^{2}\right)\right|=\ln \left|\frac{1}{\mathrm{~T}} \sum_{\mathrm{t}=1}^{\mathrm{T}} \hat{\varepsilon}_{\mathrm{t}}(\lambda) \hat{\varepsilon}_{\mathrm{t}}(\lambda)^{\prime}\right|$, siendo $\hat{\varepsilon}_{\mathrm{t}}$ el vector de residuos del modelo [2]; es decir, cuando se minimice el logaritmo del determinante de la matriz de varianzas y covarianzas del modelo TVECM recogido en [2].

Una vez obtenidos los valores estimados de los umbrales, el resto de parámetros del TVECM se pueden estimar por Mínimos Cuadrados Multivariantes. Finalmente, es necesario llevar a cabo diferentes contrastes para determinar estadísticamente si, efectivamente, el modelo no lineal estimado se ajusta mejor al mecanismo de transmisión de precios que un modelo lineal. Lo y Zivot (2001), basándose en el trabajo de Hansen (1999), proponen utilizar el siguiente estadístico: 


$$
\mathrm{LR}_{13}=\mathrm{T}\left(\ln |\hat{\Sigma}|-\ln \left|\hat{\Sigma}_{3}(\hat{\lambda})\right|\right)
$$

donde $\hat{\Sigma}$ y $\hat{\Sigma}(\hat{\lambda})$ son las matrices de varianzas y covarianzas de los modelos VECM (lineal) y $\mathrm{TVECM}_{3}$, respectivamente.

Dado que, bajo la hipótesis nula, en el modelo no lineal aparecen ciertos parámetros que no están restringidos y que no se encuentran en el modelo lineal (VECM), el estadístico [3] no sigue una distribución estándar (Davies, 1987; Hansen, 1999 y Hansen y Seo, 2002). Por tanto recurriremos a algún método de bootstrapping con el fin de obtener los valores de probabilidad del mencionado contraste.

\section{Resultados}

\subsection{Datos y propiedades estocásticas de las series}

Con el fin de analizar el mecanismo de transmisión de precios en el sector del tomate en España, se han considerado el precio percibido por el productor $\left(\mathrm{P}_{\text {Productor }}\right)$ y el precio pagado por el consumidor $\left(\mathrm{P}_{\text {Consumo }}\right.$ ), ambos expresados en $€ / \mathrm{kg}$. El período muestral se extiende desde Enero de 1986 hasta junio de 2004. El precio percibido se ha obtenido del Boletín Mensual de Estadística del Ministerio de Agricultura, Pesca y Alimentación (MAPYA), mientras que el precio al consumo se ha obtenido de la Estadísticas de Consumo Alimentario, también elaboradas por el MAPYA ${ }^{5}$. Tal como se ha comentado en el apartado 2, el precio al productor se ha multiplicado por un factor de conversión a fin de considerar productos homogéneos. Asimismo, las dos series objeto de estudio han sido transformadas en logaritmos.

Antes de proceder a la especificación y estimación del modelo adecuado para analizar el mecanismo de transmisión de precios, es necesario analizar con detalle las propiedades estocásticas de las series mediante los contrastes de raíces unitarias. Dada la notable estacionalidad que presentan las series de precios (Gráfico 1), hemos estudiado la presencia de raíces unitarias estacionales siguiendo el Método propuesto por Franses (1991) y Franses y Hobijn (1997) ${ }^{6}$. Franses (1991) propone cinco tipos de modelos para efectuar este contraste, diferenciándose en los elementos deterministas que incluyen (constante, tendencia y ficticias estaciónales). Dado que, como señalan Beaulieu y Miron (1993), la pérdida de potencia inducida por la inclusión de los elementos deterministas cuando son innecesarios es insignificante comparada con el sesgo introducido en los resultados cuando son necesarios y se omiten, en este trabajo los contrastes se han realizado incluyendo variables ficticias estacionales en el modelo a estimar lo que, por otro lado, nos permitirá determinar si la estacionalidad presente en las series, si la hay, es de naturaleza determinista o estocástica. Los resulta-

\footnotetext{
${ }^{5}$ Los autores desean agradecer a Carmen Fuentes y a la Subdirección General de Consumo Alimentario su disponibilidad a facilitarnos la información solicitada.

${ }^{6}$ Existen otros métodos de contraste de raíces estaciónales, y en particular orientados hacia series mensuales, como el de Beaulieu y Miron (1993) e Ilmakunnas (1990).
} 
dos del mencionado contraste se recogen en el Cuadro A1 del Anejo. Como se puede apreciar, los resultados confirman, para las dos series de precios, la existencia de una raíz unitaria en la frecuencia cero y un notable componente estacional determinista que puede captarse con ficticias estacionales. No se aprecia evidencia de integración en las frecuencias estacionales.

Sin embargo, como los contrastes de Franses tienen poca potencia en ausencia de integración estacional, hemos confirmado la presencia de raíces unitarias en la frecuencia regular de las series utilizando los contrastes propuestos por $\mathrm{Ng}$ y Perron (2001) quienes proponen una versión modificada de los contrastes de Phillips y Perron (1988) teniendo en cuenta, asimismo, los resultados de Elliott et al. (1996). Para las dos series consideradas en este estudio, la aplicación de los mencionados tests corroboran que ambas series son $\mathrm{I}(1)^{7}$.

\subsection{Modelo de cointegración lineal}

Dado que, como acabamos de mencionar, las series de precios no son estacionarias, el siguiente paso consiste en contrastar si dichas series están o no cointegradas utilizando el procedimiento multivariante de Johansen (1988). El modelo VAR se ha especificado con una constante restringida al espacio de cointegración ${ }^{8}$ y 11 variables ficticias estacionales. Con respecto al número óptimo de retardos del modelo VAR, en este trabajo se han utilizado dos criterios: el Criterio de Información de Akaike [Akaike (1971)] y el del Ratio de Verosimilitud propuesto por Tiao y Box (1981). Los resultados de la aplicación de ambos criterios indican que el sistema formado por las dos series de precios debería incluir cuatro retardos. En cualquier caso, se ha analizado la correcta especificación del modelo seleccionado utilizando el estadístico multivariante del Multiplicador de Lagrange de Doornik y Hendry (1997) para contrastar la presencia de autocorrelación de orden 1 y 12 (en este último caso para comprobar que efectivamente el componente estacional se capta con la introducción de 11 variables ficticias). Los resultados obtenidos de dichos estadísticos fueron de 3,03 y 28,78 , respectivamente, ambos inferiores los valores críticos correspondientes para un nivel de significación del 5\% (tal como indican los p-valores del Cuadro 1). Asimismo, se llevó a cabo el contraste de normalidad multivariante propuesto por Doornik y Hansen (1994). También en este caso, tal como se recoge en el Cuadro 1, el valor obtenido fue inferior al valor crítico al 5\%, por lo que a partir del modelo estimado se podía proceder a contrastar si los dos precios estaban cointegrados.

\footnotetext{
${ }^{7}$ Los resultados no se presentan debido a las limitaciones de espacio pero cualquier lector interesado puede solicitarlo a los autores.

${ }^{8}$ Esta elección se ha basado en el hecho de que, tanto en los análisis gráficos como en los contrastes de raíces unitarias, se ha comprobado que en todas las variables se acepta la presencia de una raíz unitaria alrededor de una media diferente de cero. Asimismo, se ha observado que los gráficos en primeras diferencias giran en torno a una media nula, lo que indica que no sería posible rechazar que $\mathrm{E}\left(\Delta \mathrm{P}_{\mathrm{f}}\right)=0$ para todos los precios. Finalmente, la constante, desde un punto de vista económico, recoge la diferencia de escala entre los dos precios considerados.
} 
En la parte superior del Cuadro 1, se recogen los resultados del estadístico de la Traza propuesto por Johansen (1988), así como sus correspondientes valores críticos. Para un nivel de significación del 5\%, los resultados obtenidos no permiten rechazar la hipótesis nula de cointegración. La relación de cointegración normalizada por el precio al consumo aparece en la segunda fila del Cuadro 1. Como se puede apreciar, la elasticidad de transmisión de precios desde el mercado en origen al mercado detallista se encuentra muy próxima a la unidad. Por tanto, el siguiente paso consiste en contrastar si la transmisión de precios es perfecta a largo plazo. El estadístico del ratio de verosimilitud para realizar dicho contraste es de 1,32 (tercera fila del Cuadro 1 ), inferior al valor crítico de una distribución $\chi^{2}$ que, para un nivel de significación del $5 \%$, es de 3,84. En consecuencia, no se puede rechazar la existencia de transmisión perfecta de precios a largo plazo en la cadena comercial o, lo que es lo mismo, cualquier variación producida en el precio en origen (detallista) se transmite en el largo plazo al precio detallista (en origen) en una variación en el mismo sentido y de la misma magnitud. La relación de equilibrio a largo plazo restringida adopta la siguiente expresión:

$$
\ln \mathrm{P}_{\text {Consumo, } \mathrm{t}}=\ln \mathrm{P}_{\text {Productor, } \mathrm{t}}-1,01=\varepsilon_{t}
$$

\section{CUADRO 1}

Resultados de los contrastes de cointegración

\begin{tabular}{|c|c|c|c|}
\hline \multirow{3}{*}{ Contraste de la Traza de Johansen } & & $\mathrm{H}_{0}: \mathrm{r}=0$ & $\mathrm{H}_{0}: \mathrm{r}=1$ \\
\hline & & 40,16 & 3,74 \\
\hline & $\mathrm{CV}(5 \%)$ & 20,16 & 9,14 \\
\hline $\begin{array}{l}\text { Estimación de la relación de } \\
\text { cointegración sin restricciones }\end{array}$ & \multicolumn{3}{|c|}{$\ln \mathrm{P}_{\text {consumo }}-0,92 \ln \mathrm{P}_{\text {productor }}-2,07=\varepsilon_{\mathrm{t}}$} \\
\hline $\begin{array}{l}\text { Contraste de homogeneidad en precios } \\
\text { a largo plazo } \mathrm{H}_{0}: \beta=(1,-1)^{\mathrm{a}}\end{array}$ & \multicolumn{2}{|c|}{$\mathrm{LR}=1,32$} & $(0,25)$ \\
\hline $\begin{array}{c}\text { Contrastes especificación multivariantes } \\
\text { Autocorrelación }{ }^{\mathrm{a}} \\
\text { No-normalidad }^{\mathrm{c}}\end{array}$ & \multicolumn{2}{|c|}{$\begin{array}{l}\text { A_LM }(1)=3,03 \\
\text { A_LM }(12)=28,78 \\
\text { N_LM }=6,39\end{array}$} & $\begin{array}{l}(0,55) \\
(0,98) \\
(0,17)\end{array}$ \\
\hline
\end{tabular}

${ }^{a}$ Los valores entre paréntesis se corresponden con los p-valores.

b A_LM(i) es el estadístico del Multiplicador de Lagrange para contrastar la hipótesis nula de ausencia de autocorrelación de orden i (Doornik y Hendry, 1997).

c N_LM es el estadístico multivariante propuesto por Doornik y Hansen (1994) para contrastar la hipótesis de normalidad. Fuente: Elaboración propia.

A partir de [4], el margen detallista a largo plazo calculado como porcentaje sobre el precio al productor viene dado por (Tiffin y Dawson, 2000):

$$
\text { Margen Detallista }=\left(\mathrm{e}^{1,01}-1\right) \times \mathrm{P}_{\text {Productor }} \times 100=175 \% \mathrm{P}_{\text {Productor }}
$$

Este resultado es consistente con lo que hemos visto en el Gráfico 2. En efecto, si tomamos el margen porcentual medio que aparece en dicha figura, el valor que 
obtenemos es del 177\%, cifra que disminuye si no consideramos el componente estacional, en cuyo caso el margen medio durante el período de estudio es del $155 \%$.

\subsection{Modelo de cointegración por umbrales}

Una vez que se ha contrastado la existencia de una relación de equilibrio a largo plazo entre el precio en origen y el precio al consumo en el sector del tomate en España, y se ha estimado dicha relación, abordaremos en los dos siguientes apartados, el objetivo fundamental de este trabajo que consiste en determinar si existen o no mecanismos de transmisión asimétricos entre los dos precios considerados. Para ello, hemos utilizado el procedimiento propuesto por Lo y Zivot (2001), descrito en el apartado anterior. El procedimiento se desarrolla en diferentes etapas. En primer lugar, se trata de determinar si el mecanismo de transmisión de precios en el sector del tomate en España se explica mejor por un modelo de cointegración lineal o por un modelo de cointegración por umbrales. Para ello, partimos de la especificación de un TVECM con dos umbrales, suponiendo conocida «a priori» la relación de equilibrio a largo plazo existente entre los dos precios. Desde este punto de vista, y con el fin de determinar si los resultados son consistentes, hemos considerado dos especificaciones alternativas de dicha relación de cointegración. La primera es la obtenida en la segunda fila del Cuadro 1, es decir la relación de cointegración lineal no restringida. La segunda es la representada en la expresión [4], esto es, la que se obtiene al imponer la restricción $(1,-1)$ sobre dicha relación de equilibrio a largo plazo. Denominaremos, TVECMU y TVECMR a los modelos no lineales que incluyan la relación de equilibrio no restringida y restringida, respectivamente. A partir de la estimación de estos dos modelos se obtienen los estimadores de los parámetros umbrales y las matrices de varianzas y covarianzas de los residuos a partir de los cuales se realizan los contrastes de no-linealidad utilizando el estadístico descrito en [3].

Los resultados de los contrastes de linealidad frente a los dos modelos con tres regímenes $\left(\mathrm{LR}_{13}\right)$ se recogen en el Cuadro 2. Como se puede observar, para un nivel de significación del 5\%, tanto para el TVEMR como para el TVECMU, el resultado del mencionado estadístico indica que se rechaza la hipótesis de linealidad en los ajustes de los precios frente a la alternativa de un modelo con dos umbrales. A continuación, se ha procedido a contrastar el número de regímenes que caracterizan el proceso de transmisión de precios en el sector del tomate en España. Para ello, se ha utilizado un estadístico similar al descrito en la expresión [3], denominado $\mathrm{LR}_{23}$, que permite contrastar la hipótesis nula de un proceso de ajuste con un único umbral (dos regímenes), frente a la alternativa de un proceso con dos umbrales (tres regímenes) ${ }^{9}$. Como se puede observar en la parte derecha del mencionado Cuadro 2, los p-valores para ambos modelos (TVECMR y TVECMU) indican que, para un nivel de significatividad del $5 \%$, no es posible rechazar la hipótesis nula de un modelo no lineal con dos regí-

\footnotetext{
${ }^{9}$ La expresión del mencionado estadístico se obtiene en la expresión [3] sustituyendo $\hat{\Sigma}$ por $\hat{\Sigma}_{2}(\hat{\lambda})$, esto es, por la matriz de varianzas y covarianzas de un modelo con dos regímenes.
} 
menes ${ }^{10}$. Los resultados de estos contrastes indican que, independientemente de la magnitud del shock, siempre se produce un proceso de ajuste en precios, lo cual es consistente con el carácter perecedero del producto en cuestión, su elevada rotación en los establecimientos detallistas y el hecho de que estamos trabajando con series mensuales (los costes de transacción pueden tener una cierta incidencia como máximo en dos o tres semanas).

Para comprobar la robustez de los resultados que acabamos de obtener, hemos comparado los mismos con el procedimiento propuesto por Hansen y Seo (2002). Dicho procedimiento permite no sólo imponer el vector de cointegración como hemos realizado hasta ahora, sino la alternativa de no imponer sus valores «a priori» y obtenerlos junto a la estimación no lineal. Adicionalmente, el enfoque de Hansen y Seo (2002) tiene la ventaja de ofrecer la estimación de un VAR completo con las dos ecuaciones bilaterales para cada uno de los dos regímenes ${ }^{11}$.

\section{CUADRO 2}

Resultados de los contrastes de no linealidad en los ajustes de precios

\begin{tabular}{lcccccc}
\hline & $\begin{array}{c}\text { Modelo lineal frente } \\
\text { a modelo no lineal con } \\
\mathbf{3} \text { regímenes }\left(\mathbf{L R}_{\mathbf{1 3}}\right)\end{array}$ & $\begin{array}{c}\text { Modelo lineal frente } \\
\text { a modelo no lineal con } \\
\mathbf{2} \text { regímenes }\left(\mathbf{L R}_{\mathbf{1 2}}\right)\end{array}$ & $\begin{array}{c}\text { Modelo no lineal con 2 } \\
\text { regímenes frente a modelo no } \\
\text { lineal con 3 regímenes }\left(\mathbf{L R}_{\mathbf{2 3}}\right)\end{array}$ \\
\cline { 2 - 7 } & $\begin{array}{c}\text { Relación de } \\
\text { equilibrio } \\
\text { restringida } \\
\boldsymbol{\beta}=\mathbf{1}\end{array}$ & $\begin{array}{c}\text { Relación de } \\
\text { equilibrio no } \\
\text { restringida } \\
\boldsymbol{\beta}=\mathbf{0 , 9 5}\end{array}$ & $\begin{array}{c}\text { Relación de } \\
\text { equilibrio } \\
\text { restringida } \\
\boldsymbol{\beta}=\mathbf{1}\end{array}$ & $\begin{array}{c}\text { Relación de } \\
\text { equilibrio no } \\
\text { restringida } \\
\boldsymbol{\beta}=\mathbf{0 , 9 5}\end{array}$ & $\begin{array}{c}\text { Relación de } \\
\text { equilibrio } \\
\text { restringida } \\
\boldsymbol{\beta}=\mathbf{1}\end{array}$ & $\begin{array}{c}\text { Relación de } \\
\text { equilibrio no } \\
\text { restringida } \\
\boldsymbol{\beta}=\mathbf{0 , 9 5}\end{array}$ \\
\hline Estadístico & 79,98 & 81,09 & 48,97 & 41,59 & 38,32 & 33,87 \\
P-valor & 0,041 & 0,039 & 0,036 & 0,040 & 0,13 & 0,18 \\
\hline
\end{tabular}

a Los p valores se han obtenido mediante la técnica de bootstrapping de regresores fijos (Hansen, 1999; y Hansen y Seo, 2002).

Fuente: Elaboración propia.

Estos autores proponen dos estadísticos LR para contrastar si se ajusta mejor a los datos un modelo de cointegración lineal frente a la alternativa no lineal. El primer contraste está basado en que el valor del vector de cointegración es conocido a priori $(\beta=1)$ (equivalente al contraste sobre el modelo TVECMR del Cuadro 2), mientras que en el segundo se supone que el verdadero valor del vector de cointegración no es conocido a priori y debe ser estimado simultáneamente junto con el valor del parámetro umbral (equivalente al contraste sobre el modelo TVECMU del

${ }^{10}$ A fin de completar el panorama, en el Cuadro 3 también se presentan los resultados del contraste de la hipótesis nula de un modelo lineal frente al TVECM 2 . Como se puede apreciar, también en este caso el modelo no lineal es preferido al lineal.

11 Agradecemos a un revisor anónimo la sugerencia en este sentido. 
Cuadro 2) ${ }^{12}$. Los resultados de los contrastes de Hansen y Seo (2002) aparecen en el Cuadro 3.

\section{CUADRO 3}

Contrastes de cointegración umbral de Hansen y Seo (2002)

\begin{tabular}{|l|c|c|}
\hline & $\boldsymbol{\beta}$ restringido & $\boldsymbol{\beta}$ no restringido \\
\hline Estadístico Sup LM (p-valor) & $49,398(0,033)$ & $47,10(0,037)$ \\
\hline Vector de cointegración estimado & $(1,-1)$ & $(1,-0,93)$ \\
\hline Parámetro umbral (1) & $-0,073$ & $-0,053$ \\
\hline Porcentaje de observaciones en cada régimen & $52 \%$ (régimen 1) & $\begin{array}{l}46 \% \text { (régimen 1) } \\
48 \% \text { (régimen 2) }\end{array}$ \\
\hline $\begin{array}{l}\text { Estadístico Wald para contrastar la igualdad de los } 2) \\
\text { parámetros a corto plazo entre los dos regímenes } \\
\text { (p valor) }\end{array}$ & $174,76(0,00)$ & $175,159(0,00)$ \\
\hline $\begin{array}{l}\text { Estadístico Wald para contrastar la igualdad de los } \\
\text { coeficientes de corrección del error entre los dos } \\
\text { regímenes (p valor) }\end{array}$ & $21,37(0,00)$ & $18,72(0,00)$ \\
\hline
\end{tabular}

Fuente: Elaboración propia.

Tanto en el caso de que $\beta$ sea fijado en la unidad, como en el que no se encuentre restringido, los valores asintóticos calculados a partir de los métodos de simulación, rechazan la hipótesis nula de cointegración lineal al 5\% a favor de la alternativa del modelo de corrección del error no lineal. Asimismo, los resultados de los contrastes de Wald (recogidos en las dos últimas filas del Cuadro 3) confirman estos resultados ya que se rechazan las hipótesis nulas de igualdad de los coeficientes de corrección del error y de los parámetros de corto plazo entre los dos regímenes, respectivamente. El parámetro de cointegración estimado es que, como se puede observar, es muy similar al obtenido en el Cuadro 2. En definitiva, los resultados obtenidos por los dos procedimientos pueden considerarse como muy robustos. Por otro lado, teniendo en cuenta que el parámetro estimado de la relación de equilibrio a largo plazo se encuentra muy próximo a la unidad, y los resultados de los contrastes efectuados en la sección anterior, en el resto del trabajo hemos considerado únicamente el modelo con el parámetro de largo plazo restringido a la unidad.

En la tercera fila del Cuadro 3 se recoge el valor del umbral estimado para el modelo con b fijado en la unidad $(-0,073)$. Este valor umbral o coste de transacción obtenido se puede considerar como un estimador de la distorsión existente en la trans-

12 Al igual que en el procedimiento de Lo y Zivot (2001), la metodología de Hansen y Seo (2002) estima simultáneamente los dos parámetros, el de cointegración $(\hat{\beta})$ y el parámetro umbral $(\hat{\lambda})$ minimizando la expresión: $(\hat{\lambda}, \hat{\beta})=\arg \min \left[\hat{\Sigma}_{2}(\lambda, \beta)\right]$ donde $\hat{\Sigma}_{2}(\lambda, \beta)$ es la matriz estimada de varianzas y covarianzas

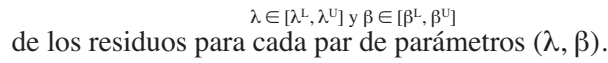


misión de precios a lo largo de la cadena de comercialización del sector del tomate en España. Desgraciadamente, todavía no existe un procedimiento validado en la literatura para realizar inferencia estadística sobre el estimador del parámetro umbral, dado que dicho parámetro posee una distribución limite no estándar que depende de los momentos de la muestra (Hansen, 1997). No obstante, Chan (1993) y Chan y Tsay (1998) demostraron que los parámetros umbrales son superconsistentes y, por tanto, sus estimadores pueden ser considerados como los verdaderos valores en las aplicaciones empíricas.

A partir de la magnitud del umbral estimado, se pueden diferenciar dos regímenes diferentes en el proceso de transmisión de precios en el sector del tomate en España. El primer régimen se asocia a situaciones en las que, según las condiciones del mercado, el margen comercial del detallista se sitúa por debajo del del precio al productor, mientras que el segundo régimen aparece asociado a la existencia de un margen comercial por encima del $155 \%$ del precio al productor.

Una vez que se ha establecido el parámetro umbral, el resto de los parámetros del TVECM se pueden estimar por MCO multivariantes:

$$
\Delta P_{t}=\left(\begin{array}{l}
\Delta P C_{t} \\
\Delta P P_{t}
\end{array}\right)=\sum_{i=1}^{11} \delta_{i} D_{i t}+\left\{\begin{array}{l}
\left(\begin{array}{l}
\alpha_{1}^{1} \\
\alpha_{2}^{1}
\end{array}\right) \omega_{t-1}(\beta)+\sum_{i=1}^{3} \Gamma_{i}^{1} \Delta \mathrm{P}_{t-i}+\varepsilon_{t}^{1}, \text { si } \omega_{t-1}(\beta) \leq-0,073 \\
\left(\begin{array}{l}
\alpha_{1}^{2} \\
\alpha_{2}^{2}
\end{array}\right) \omega_{t-1}(\beta)+\sum_{i=1}^{3} \Gamma_{i}^{2} \Delta \mathrm{P}_{t-i}+\varepsilon_{t}^{2}, \text { si } \omega_{t-1}(\beta)>-0,073
\end{array}\right.
$$

donde: $\omega_{t-1}(\beta)=\ln \mathrm{P}_{\text {Consumo } t-1}-\ln \mathrm{P}_{\text {Productor } t-1}-1,01$

En el Cuadro 4 se recogen los principales parámetros estimados del modelo [6] que permiten explicar la dinámica de ajuste de los precios. Asimismo, se han incluido las estimaciones de los parámetros de ajuste $\left(\hat{\rho}_{i}\right)^{13}$, que miden la velocidad con la que el vector de cointegración regresa a su valor medio después de haberse producido un shock en el sistema. Como se puede observar, el parámetro de ajuste $\hat{\rho}_{i}$ en el primer régimen $(0,52)$ es inferior al estimado para el segundo régimen $(0,67)$. Este resultado indica que el ajuste hacia el equilibrio después de que se produzca un shock es más rápido cuando el margen comercial disminuye que cuando aumenta. La velocidad de ajuste se mide por lo que se denomina Vida Media $\left[\ln (0,5) / \ln \left(\hat{\rho}_{i}\right)\right]$ que no es sino el número de períodos que se requiere para reducir en la mitad la desviación que se ha producido, después del shock, con respecto al equilibrio a largo plazo (Obstfeld and Taylor, 1997). En el primer régimen la Vida Media se estima en algo más de un mes mientras que durante el segundo régimen es de, aproximadamente, 1,75 meses.

${ }^{13}$ El coeficiente de ajuste viene definido por: $\rho^{\mathrm{j}}=1+\beta^{\prime} \alpha^{\mathrm{j}}=1+\left[1-\beta_{2}\right]\left[\begin{array}{l}\alpha_{1}^{\mathrm{j}} \\ \alpha_{2}^{\mathrm{j}}\end{array}\right]=1+\alpha_{1}^{\mathrm{j}}-\beta_{2} \alpha_{2}^{\mathrm{j}}$ 


\section{CUADRO 4}

Estimación de los parámetros del modelo de cointegración por umbrales ${ }^{\mathrm{a}}$

\begin{tabular}{|c|c|c|c|c|c|}
\hline \multicolumn{3}{|c|}{$\begin{array}{c}\text { Régimen 1 } \\
\ln P_{\text {consumo }}-\ln P_{\text {productor }}-1,01 \leq-0,073\end{array}$} & \multicolumn{3}{|c|}{$\begin{array}{c}\text { Régimen 2 } \\
\ln P_{\text {consumo }}-\ln P_{\text {productor }}-1,01>-0,073 \text { b }\end{array}$} \\
\hline$\left(\begin{array}{l}\alpha_{c}^{1} \\
\alpha_{p}^{1}\end{array}\right)$ & $\hat{\rho}^{\mathrm{b}}$ & Vida Media ${ }^{c}$ & $\left.\begin{array}{l}\alpha_{c}^{2} \\
\alpha_{p}^{2}\end{array}\right)$ & $\hat{\rho}^{\mathrm{b}}$ & Vida media $^{c}$ \\
\hline$\left(\begin{array}{c}-0,18 \\
(-2,17) \\
-0,389 \\
(2,072)\end{array}\right)$ & 0,52 & 1,05 meses & $\left(\begin{array}{c}-0,031 \\
(-0,625) \\
0,628 \\
(2,981)\end{array}\right)$ & 0,67 & 1,73 meses \\
\hline
\end{tabular}

a Los valores entre paréntesis se corresponden con los t-ratios.

b $\rho$ es el coeficiente de ajuste y representa la velocidad a la cual el vector de cointegración regresa al valor medio de cada régimen.

c La vida media se define como $\left[\ln (0,5) / \ln \left(\hat{\rho}_{\mathrm{i}}\right)\right]$.

Fuente: Elaboración propia.

En todo caso, los parámetros realmente relevantes en los modelos de cointegración por umbrales son los coeficientes estimados de la matriz a asociados a la relación de equilibrio $\omega_{t-1}(\beta)$. El signo y la magnitud de estos coeficientes nos permiten analizar cómo es el proceso de ajuste temporal por el que se restaura el equilibrio a largo plazo entre ambos precios. Como se puede apreciar en el Cuadro 4, los cuatro coeficientes de la matriz $\alpha$ son significativos salvo el correspondiente al precio al consumo en el segundo régimen (aumento de márgenes). Para ver las implicaciones de este resultado, el Gráfico 3 muestra cuál sería el comportamiento de ambos precios en los dos regímenes considerados. En el primer régimen, los dos coeficientes de ajuste son significativos. Teniendo en cuenta los signos de la relación [4] y los de los parámetros de ajuste, los resultados indican que ambos precios regresarían a su nivel de equilibrio después de un shock. Una reducción del margen puede ser debida a un incremento del precio al productor o un aumento del precio al consumo (figuras izquierda y derecha, respectivamente). Si observamos el gráfico de la izquierda, tras el shock inicial en el precio al productor, el precio al consumo reacciona en un momento posterior de tal forma que se re-establece el equilibrio a largo plazo. En el caso de que se produzca una disminución del precio al consumo, el precio al productor reacciona a la baja después de un cierto tiempo para volver a recuperar el margen de equilibrio a largo plazo.

Consideremos, a continuación, una fase de crecimiento del margen comercial que puede ser provocado bien por un aumento del precio al consumo, bien por una disminución del precio al productor (figuras derecha e izquierda de la parte inferior del Gráfico 3). Si el precio al consumo aumenta, tras un período de ajuste, el precio del productor aumenta para retornar al equilibrio a largo plazo. Sin embargo, al disminuir el precio al productor los precios al consumo no reaccionan ( $\alpha_{c}^{2}$ no es significativo), lo que genera un incremento del margen, en beneficio del sector detallista. En definitiva, lo que se deduce es que los precios al consumo son mucho más rígidos cuando 


\section{GRÁFICO 3}

Ajustes de los precios según el modelo TVECM $_{2}$ estimado

Primer régimen: Reducción del margen

Incremento del precio al productor

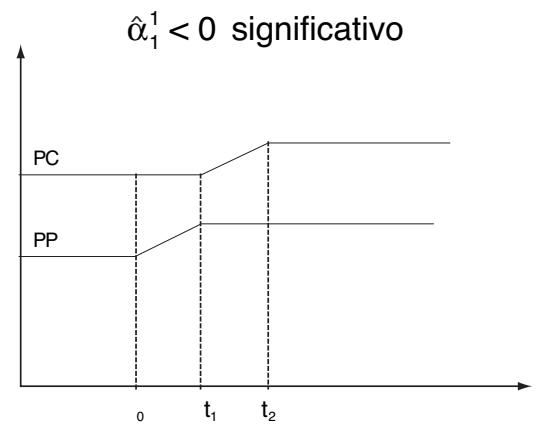

Disminución del precio al consumo

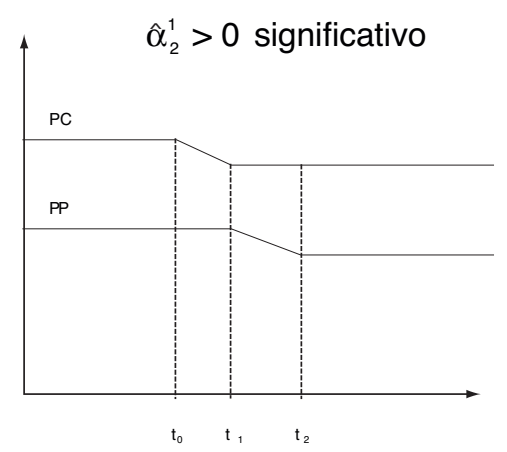

Segundo régimen: Ampliación del margen
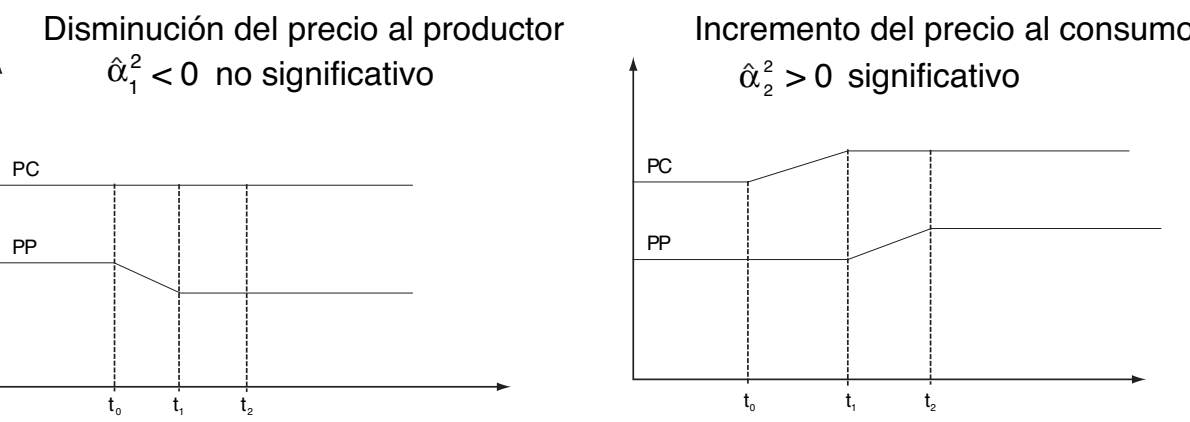

Fuente: Elaboración propia.

los precios al productor disminuyen que cuando estos aumentan. Este resultado es consistente con una gran parte de la literatura existente sobre el tema, especialmente los trabajos de Ben-Kaabia y Gil (2007), von Cramon-Taubadel (1998) y Abdulai (2002). Finalmente, teniendo en cuenta la magnitud de los coeficientes de ajuste, podemos también afirmar que tanto el precio al consumo como el del productor reaccionan más rápidamente cuando el margen comercial aumenta (como consecuencia de un aumento del precio al consumo) que cuando éste se reduce (disminución del precio al consumo).

A pesar de que los resultados obtenidos en el Cuadro 4 nos dan una idea aproximada de las asimetrías existentes en el mecanismo de transmisión de precios en el sector del tomate en España, el análisis de la dinámica del corto plazo nos va a permitir determinar con mayor precisión la magnitud y dirección de dichas relaciones asimétricas. A dicho análisis dedicaremos el próximo apartado. 


\subsection{Análisis de la dinámica de ajuste a corto plazo}

En los modelos TVECM el análisis de la dinámica a corto plazo suele realizarse a partir del cálculo de las denominadas Funciones Impulso-Respuesta No lineales (FIRN) [Koop et al. (1996) y Potter (1995)]. La FIRN ante un shock y un valor arbitrario de $\mathrm{P}_{\mathrm{t}-1}=\mathrm{j}_{\mathrm{t}-1}$ (denominado como la historia o el pasado del sistema) viene dado por la siguiente expresión:

$$
\begin{aligned}
\operatorname{FIRN}\left(\mathrm{n}, \delta, \varphi_{\mathrm{t}-1}\right)= & \mathrm{E}\left[\mathrm{P}_{\mathrm{t}+\mathrm{n}} \mid \varepsilon_{\mathrm{t}}=\delta, \varepsilon_{\mathrm{t}+1}=\ldots=\varepsilon_{\mathrm{t}+\mathrm{n}}=0, \varphi_{\mathrm{t}-1}\right] \\
& -\mathrm{E}\left[\mathrm{P}_{\mathrm{t}+\mathrm{n}} \mid \varepsilon_{\mathrm{t}}=0, \varepsilon_{\mathrm{t}+1}=\ldots=\varepsilon_{\mathrm{t}+\mathrm{n}}=0, \varphi_{\mathrm{t}-1}\right] \mathrm{n}=0,1, \ldots \mathrm{N}
\end{aligned}
$$

Por tanto, las FIRN se calculan como la diferencia entre dos realizaciones de la serie $\mathrm{P}_{\mathrm{t}}$ teniendo en cuenta el conjunto de información disponible hasta el período $\mathrm{t}-1$. La primera es la realización de $\mathrm{P}_{\mathrm{t}}$ después que haya ocurrido un shock $\varepsilon_{\mathrm{t}}=\delta$ en el instante $\mathrm{t}$, mientras que la segunda realización es el valor de $\mathrm{P}_{\mathrm{t}}$ que se obtendría si no hubiera ocurrido ningún shock. Teniendo en cuenta esta definición, las FIRN varían en función de los valores que toman $\delta \in \varepsilon_{\mathrm{t}}$ y $\varphi_{\mathrm{t}-1} \in \Omega_{\mathrm{t}-1}\left(\Omega_{\mathrm{t}-1}\right.$ es el conjunto de información disponible en el instante $\mathrm{t}-1$ utilizado para predecir los valores futuros de $\mathrm{P}_{\mathrm{t}}$ ). A partir de esta definición se pueden analizar las respuestas de los precios al consumidor (productor) ante un shock positivo (negativo) en los precios en origen (consumo) en cada uno de los regímenes identificados. Asimismo, las FIRN se pueden utilizar para evaluar el nivel de significatividad de los ajustes asimétricos a lo largo de los lapsos temporales. En este sentido, Potter (1995) definió una medida para evaluar el nivel de asimetría de las respuestas como la suma de las FIRN para un determinado shock y las FIRN para un shock de la misma magnitud pero con el signo opuesto. Dicha medida viene dada por la siguiente expresión:

$$
\operatorname{ASY}\left(\mathrm{n}, \varepsilon_{\mathrm{t}}, \varphi_{\mathrm{t}-1}\right)=\operatorname{FIRN}\left(\mathrm{n},+\delta_{\mathrm{i}}, \varphi_{\mathrm{t}-1}\right)+\operatorname{FRIN}\left(\mathrm{n},-\delta_{\mathrm{i}}, \varphi_{\mathrm{t}-1}\right)
$$

Finalmente, para determinar el nivel de significatividad tanto de las funciones impulso respuesta como de la medida de asimetría se pueden generar intervalos de confianza utilizando las técnicas de bootstrapping (Franses y van Dijk, 2000). En este estudio, para cada respuesta y para los coeficientes de asimetría se han estimado los correspondientes intervalos de confianza al $95 \%$ basadas en 5.000 replicaciones ${ }^{14}$. Las respuestas significativas se han marcado con un recuadro en negro.

En la parte izquierda del Gráfico 4 se recogen las respuestas del precio al consumo y del precio al productor como consecuencia de un shock en éste último. En la parte derecha se recoge la medida propuesta por Potter (1995) para evaluar la asimetría de las respuestas obtenidas. Como se puede observar, en un entorno de reducción de márgenes comerciales, un shock positivo en el precio al productor

14 Todos los análisis han sido programados en GAUSS. 


\section{GRÁFICO 4}

Funciones impulso respuesta a un shock del $\pm 1 \%$ en el precio al productor

Shock en el precio al productor

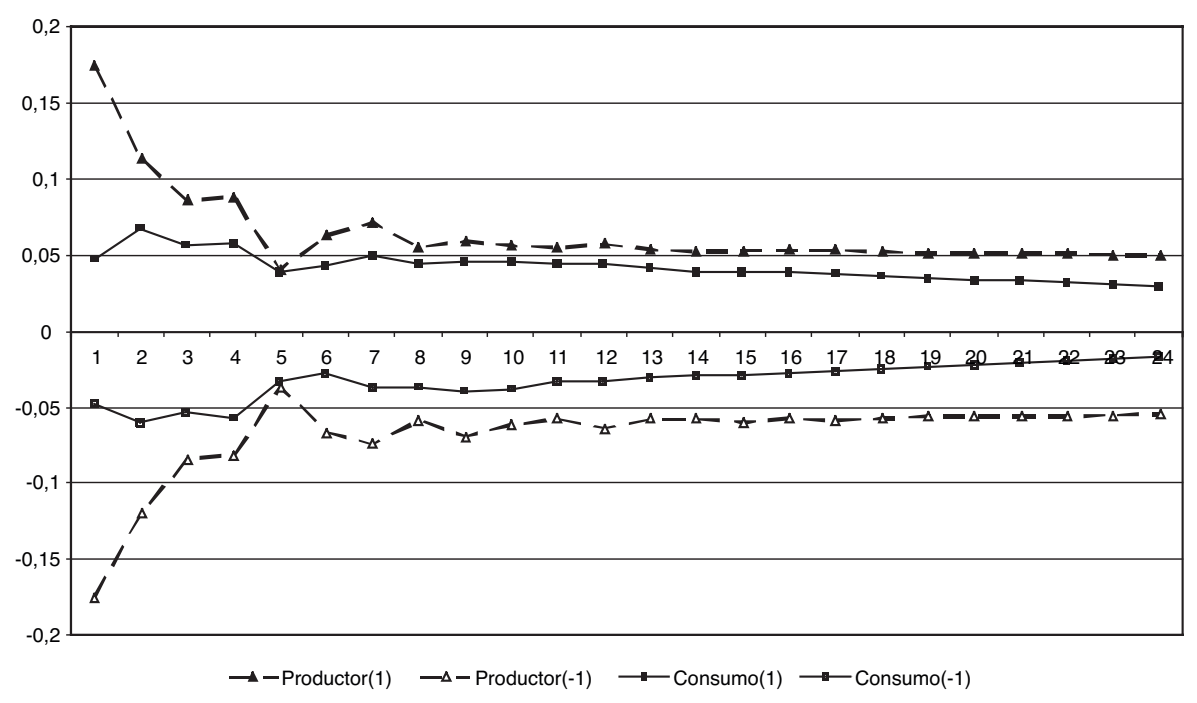

Shock en el precio al productor

Respuestas asimétricas acumuladas

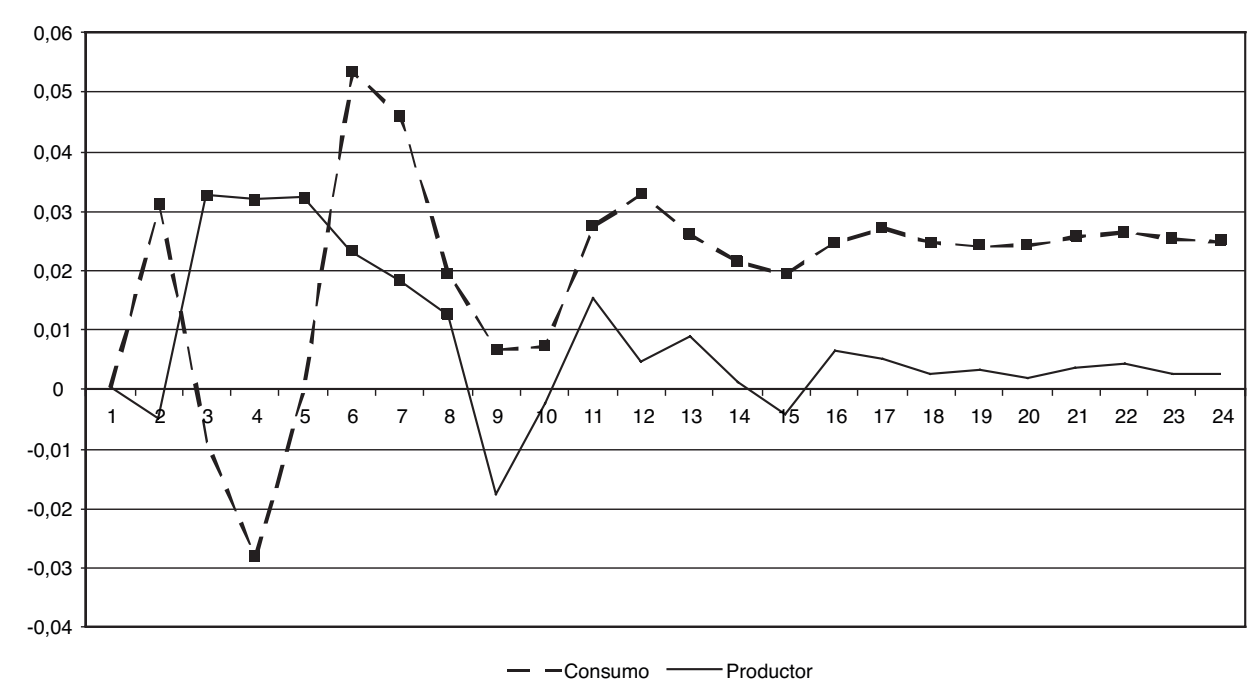

Nota: Un * en el gráfico de la derecha indica que la respuesta es significativamente diferente de cero para un nivel de significación del 5\%.

Fuente: Elaboración propia. 
tiende a reducir todavía más dicho margen, al menos durante los meses inmediatamente posteriores al shock. En efecto, la respuesta del precio al productor es más del triple que la del detallista durante el primer mes. Durante el segundo mes, el margen se reduce significativamente como consecuencia de un ligero aumento del precio al consumo y una disminución del precio al productor. A partir del tercer mes, las respuestas de los dos precios no son estadísticamente diferentes entre sí. Este comportamiento puede explicarse por el carácter perecedero del producto objeto de estudio. En un entorno de precios percibidos al alza los detallistas están dispuestos a sacrificar su margen antes de quedarse con excedentes no vendidos.

En todo caso, y hasta cierto punto, esta situación se compensa en situaciones de exceso de oferta. Como puede observarse en el Gráfico 4, un descenso en el precio al productor como consecuencia de un exceso de oferta genera un aumento del margen comercial. El patrón de comportamiento de las respuestas de ambos precios es, aparentemente, bastante similar al que acabamos de comentar cuando se producía un shock positivo en el precio al productor. En efecto, la respuesta a muy corto plazo del precio percibido es de mayor magnitud que la experimentada por el precio al consumo, tal como ocurría en el caso anterior. Sin embargo, a medida que discurre el tiempo, el diferencial de respuestas es mayor que en el caso de un shock positivo, siempre a favor del sector detallista.

Por tanto, y aunque, aparentemente, de la parte izquierda del Gráfico 4 pueda desprenderse que el proceso de transmisión es simétrico, la parte derecha nos arroja un resultado completamente distinto. A la vista del mencionado gráfico se pueden obtener dos conclusiones. La primera es que tanto productores como detallistas se benefician de asimetrías positivas, es decir, las reacciones son de superior magnitud cuando se trata de shocks positivos que cuando se trata de shocks negativos. La segunda conclusión es que mientras los beneficios de los productores se realizan en el corto plazo (las asimetrías sólo son significativamente diferentes de cero entre el tercer y el octavo mes después del shock), los detallistas se benefician a largo plazo. En efecto, a partir del quinto y a lo largo de todos los horizontes de predicción, las asimetrías positivas son significativas. Este resultado permite concluir que los procesos inflacionistas originados en la cadena de valor, en el caso de los productos agroalimentarios perecederos, no son debidos al aumento del valor del producto a pie de explotación sino que se deben a incrementos de los márgenes comerciales.

Analicemos, para finalizar, las respuestas de ambos precios a un shock del 1\%, tanto positivo como negativo, del precio al consumo (Gráfico 5). La conclusión a la que llegamos es prácticamente la misma que la obtenida en el caso anterior, si bien la magnitud de las respuestas sigue un patrón totalmente diferente. En efecto, un shock positivo en el precio al consumo genera una respuesta, también positiva, tanto del precio al productor como del precio al consumo. Además, en el corto plazo, la magnitud de las respuestas es casi idéntica mientras que, a partir del quinto mes, se produce un diferencial, a favor del precio al consumo, que se mantiene constante a lo largo del tiempo. En cambio, las respuestas ante un shock negativo son prácticamente idénticas a lo largo de todo el horizonte de predicción. 


\section{GRÁFICO 5}

Funciones impulso respuesta a un shock del $\pm 1 \%$ en el precio al consumo

Shock en el precio al consumo

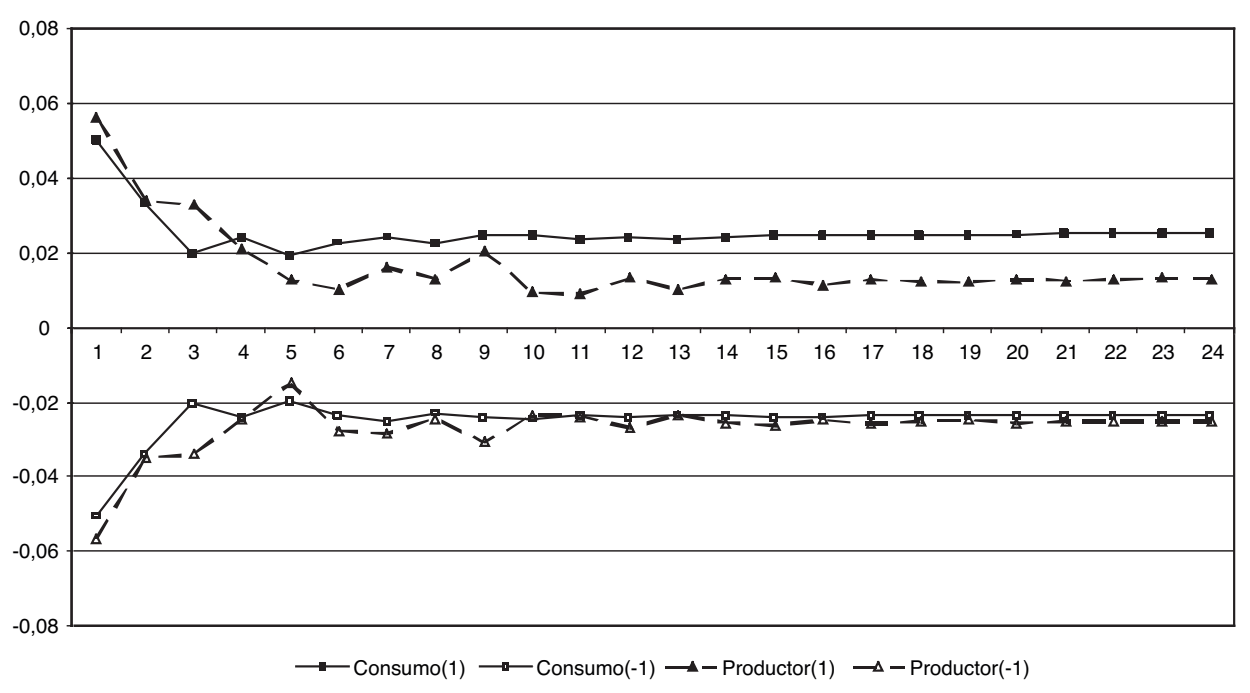

Shock en el precio al consumo

Respuestas asimétricas acumuladas

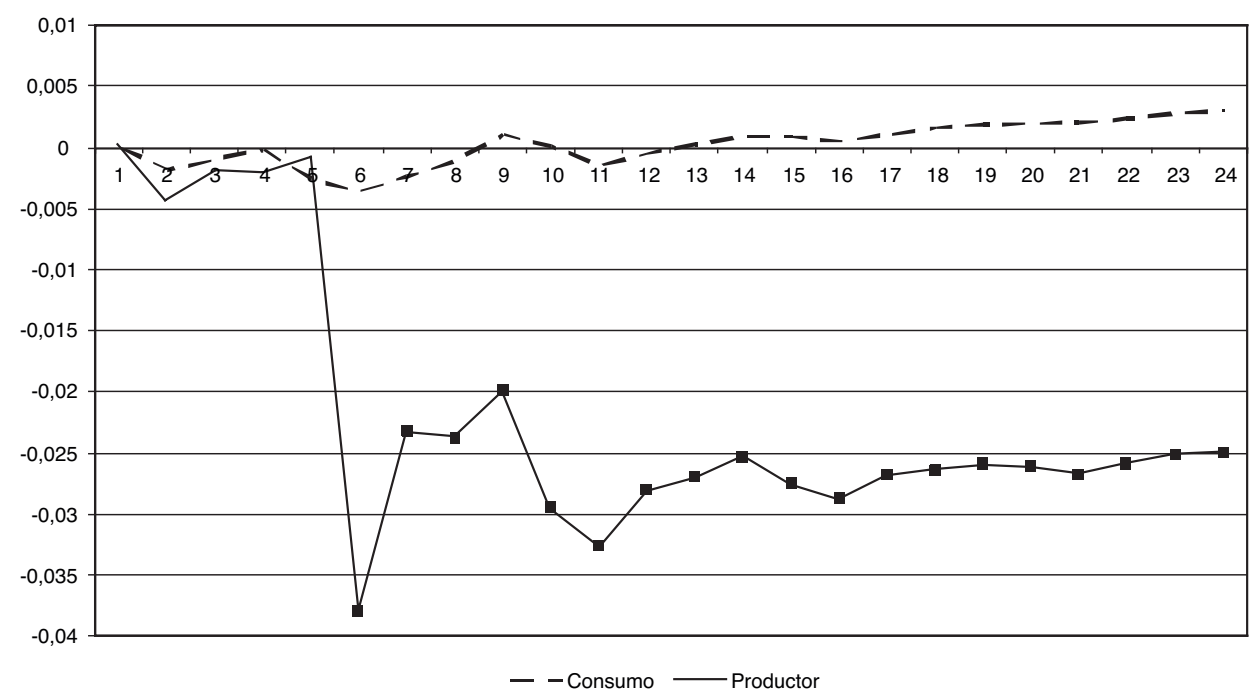

Nota: Un * en el gráfico de la derecha indica que la respuesta es significativamente diferente de cero para un nivel de significación del 5\%.

Fuente: Elaboración propia. 
El resultado de estos dos comportamientos se resume en la parte derecha del Gráfico 5. Como se puede apreciar, las respuestas del precio al consumo se pueden considerar simétricas. Las respuestas ante shocks positivos y negativos no difieren significativamente en magnitud. Sin embargo, las respuestas del precio percibido son asimétricamente negativas. Esto es, cuando los precios al consumo aumentan, los precios al productor aumentan en una proporción similar. Sin embargo, cuando disminuyen, el sector detallista presiona al sector productor y el precio percibido disminuye en mayor proporción, obteniéndose como resultado un aumento del margen comercial.

\section{Consideraciones finales}

El análisis de las relaciones de precios a lo largo de la cadena comercial nos permite aproximarnos al funcionamiento de los mercados. En este trabajo hemos aplicado la metodología de la cointegración por umbrales para caracterizar los procesos de ajuste de los precios en el sector del tomate en España ante cambios en las condiciones de oferta y demanda en los diferentes escalones de la cadena comercial. Si bien no podemos relacionar de forma directa la existencia de asimetrías con mercados ineficientes, no es menos cierto que respuestas rápidas y simétricas suelen caracterizar a los mercados eficientes.

Los resultados obtenidos en este trabajo sugieren que en el largo plazo los precios son homogéneos, esto es, cualquier variación en uno de ellos genera una reacción de la misma magnitud del otro precio de tal forma que el margen se mantiene relativamente estable. La duración completa del ajuste estaría en unos cinco meses. Este resultado ha sido corroborado por la simple inspección gráfica del margen comercial relativo, es decir, en relación con el precio percibido. Dicho margen se ha mantenido más o menos estable en torno al 150 y $175 \%$. No era el objetivo de este trabajo determinar si este margen es excesivo ya que nos disponemos en España de información (fiable o no) de la estructura de costes, no sólo a nivel de explotación (que sí la hay) sino a lo largo de la cadena de valor. Desde este punto de vista, todavía queda mucha investigación por hacer ya que la única información disponible es la relativa a los precios (destaquemos aquí el esfuerzo realizado por el Ministerio de Agricultura en relación al Observatorio de precios).

A pesar de que en el largo plazo, existe una perfecta integración de los precios, se aprecia que en el corto plazo los detallistas siempre se benefician de cualquier shock de oferta o demanda en los diferentes escalones de la cadena comercial. Tanto si varían las condiciones de oferta primaria (variaciones de los precios al productor) como de demanda derivada (variaciones en el precio al consumo), el margen comercial aumenta, siendo el responsable de los procesos inflacionistas que tradicionalmente han caracterizado a los productos perecederos.

\section{Bibliografía}

Abdulai, A. (2002). «Using threshold cointegration to estimate asymmetric price transmission in the Swiss pork market». Applied Economics, 34:679-687. 
Akaike, H. (1971). «Fitting autoregressive models for prediction.» Annals of the Institute of Statistical Mathematics, 21:243-247.

Andrews, D.W.K. (1993). «Tests for parameter instability and structural change with unknown change point». Econometrica, 61(4):821-856.

Azzam, A. (1999). «Asymmetry and rigidity in farm-retail price transmission». American Journal of Agricultural Economics, 81:525-533.

Bailay, D.V. y Brorsen, B.W. (1989). «Price asymmetric in spatial fed cattle markets.» Western Journal of Agricultural Economics, 14:246-252.

Balke, N.S. y Fomby, T.S. (1997). «Threshold cointegration». International Economic Review, 38:627-645.

Beaulieu, J.J. y Miron, J.A. (1993). «Seasonal unit roots in aggregate U.S. data». Journal of Econometrics, 55:305-328.

Ben-Kaabia, M. y Gil, J.M. (2007).»Asymmetric price transmission in the Spanish lamb sector». European Review of Agricultural Economics, 34(1):53-80.

Chan, K.S. (1993). «Consistency and limiting distribution of the least squares estimator of a threshold autoregressive model». The Annals of Statistics, 21:520-533.

Chan, K.S. y Tsay, R.S. (1998) «Limiting properties of the least squares estimator of a continuous threshold autoregressive model», Biometrika, 85(2):413-426,

Chavas, J.P. y Metha, A. (2004). «Price dynamics in a vertical sector: the case of butter». American Journal of Agricultural Economics, 86:1078-1093.

Davies, R.B. (1987). «Hypothesis testing when a nuisance parameter is present only under the alternative». Biometrika, 74:33-43.

Doornik, J.A. y Hansen, H. (1994). A practical test of multivariate normality, unpublished paper, Nuffield College.

Doornik, J.A. y Hendry, D.F. (1997). Modelling dynamic systems using PcFilm 9 for Windows. Timberlake Consulting, London.

Elliott, G., Rothenberg, T.J. y Stock, J.H. (1996). «Efficient tests for an autoregressive unit root». Econometrica, 64:813-836.

Enders, R.F. y Granger, C.W.J. (1998). «Unit root tests and asymmetric adjustment with an example using the term structure of interest rates.» Journal of Business and Economic Statistics, 16:304-312.

Engle, R.F. y Granger, C.W.J. (1987). «Co-integration and error correction: representation, estimation and testing». Econometrica 55:251-276.

Franses, P.H. (1991). Model selection and seasonality in time series. Tinbergen Institute series n. ${ }^{\circ} 18$. Erasmus University. Roterdam.

Franses, P.H. y Hobijn, B. (1997). «Critical values for unit root tests in seasonal time series». Journal of Applied Statistics, 24:25-46

Franses, P.H. y van Dijk, D. (2000). Non-linear time series models in empirical finance. Cambridge University Press.

Frost, D. y Bowden, R. (1999). «An asymmetry generator for error-correction mechanisms, with application to bank mortgage-rate dynamics». Journal of Business and Economic Statistics, 17(2):253-263.

Goodwin, B.K. y Piggott, N.E. (2001). «Spatial market integration in the presence of threshold effects». American Journal of Agricultural Economics, 83(2):302-317

Gonnzalo, J. y Pitarakis, J. (2006). «Threshold effects in multivariate error correction models». En Mills, T.C. y Patterson, K. (eds) Palgrave Handbook of Econometrics, Vol. I, Chapter 15:578-609. 
Granger, C.W.J. y Lee, T.H. (1989). «Investigation of production, sales and inventory relationships using multicointegration and non-symmetric error correction models». Journal of Applied Econometrics, 2:111-120.

Griffith, G.R. y Piggot, N.E. (1994). «Asymmetry in beef, lamb and pork farm-retail price transmission in Australia». Agricultural Economics, 10:307-316

Hansen, B.E. (1997). «Inference in TAR models». Studies in Nonlinear Dynamics and Econometrics, 2:1-14.

Hansen, B.E. (1999). «Testing for linearity». Journal of Economic Surveys, 13, 551-576.

Hansen, B.E. y Seo, B. (2002). «Testing for two-regime threshold cointegration in vector error correction models». Journal of Econometrics, 110:293-318.

Houck, J.P. (1977). «An approach to specifying and estimating nonreversible functions». American Journal of Agricultural Economics, 59:570-572.

Ilmakunnas, P. (1990). «Testing the order of differencing in quarterly data: an illustration of the testing sequence». Oxford Bulletin of Economics and Statistics, 52:79-88.

Johansen, S. (1988). «Statistical analysis of the cointegration vectors». Journal of Economic Dynamics and Control, 12:231-254.

Kinnucan, H.W. y Forker, O.D. (1987). «Asymmetry in the farm-retail price transmission for major dairy products». American Journal of Agricultural Economics, 69:285-292.

Koop, G., Pesaran, M.H. y Potter, S.M. (1996). «Impulse response analysis in nonlinear multivariate models». Journal of Econometrics, 74:119-147.

Lo, C. y Zivot, E. (2001). «Threshold cointegration and nonlinear adjustments to the law of one price». Macroeconomic Dynamics, 5:533-576.

MAPA (varios años). Boletín mensual de estadística. Secretaria General Técnica, Madrid.

MAPA (2006). Anuario de estadídtica agraria, 2004. Secretaria General Técnica, Madrid.

McCorriston, S., Morgan, C.W. y Rayner, A.J. (2001). «Price transmission: The interaction between market power and returns to scale». European Review of Agricultural Economics, 28:143-159.

Meyer, J. y von Cramon-Taubadel, S. (2004). «Asymmetric price transmission: A survey». Journal of Agricultural Economics, 55(3):581-611.

Ng, S. y Perron, P. (2001). «Lag length selection and the construction of unit root tests with good size and power». Econometrica, 69:1519-1554.

Obstfeld, M. y Taylor, A. M. (1997). «Non-linear aspects of goods-market arbitrage and adjustment: Heckscher's commodity points revisited.» The Journal of the Japanese and International Economics, 11:441-479.

Phillips P.C.B. y Perron, P. (1988). «Testing for a unit root in time series regression». Biometrika, 75:335-346.

Potter, S.M. (1995). «A nonlinear approach to U.S. GNP.» Journal of Applied Economics, 10:109-125.

Reagan, P.B. y Weitzman, M.L. (1982). «Asymmetries in price and quantity adjustments by the competitive firm». Journal of Economic Theory, 27:410-420

Sanjuán, A.I., Gil, J.M. y Albisu, L.M. (1996). Una aproximación a la medición de la incidencia de los precios agrarios en los precios al consumo de alimentos (1987-1992). Monografías INIA, 94. Instituto Nacional de Investigación y Tecnología Agraria y Alimentaria, Madrid.

Serra, T. y Goodwin, B.K. (2002). «Specification selection issues in multivariate threshold and switching Models.» Paper presented at the AAEA-WAEA Annual Meeting

Tiao, G.C. y Box, G.E. (1981). «Modeling multiple time series applications.» Journal of American Statistical Association, 76:802-816. 
Tiffin, R. y Dawson, P.J. (2000). «Structural break, cointegration and the farm-retail price spread for lamb». Applied Economics, 32:1281-1286.

Tsay, R. (1998). «Testing and modelling multivariate threshold models». Journal of the American Statistical Association, 93(1):1188-1202.

von Cramon-Taubadel, S. (1998). «Estimating asymmetric price transmission with the error correction representation: An application to the German pork market». European Review of Agricultural Economics, 25:1-18.

Ward, R.W. (1982). «Asymmetry in retail, wholesale and shipping point pricing for fresh vegetable». American Journal of Agricultural Economics, 64:205-212.

Wolffram, R. (1971). «Positivistic measures of aggregate supply elasticities: some new approaches - Some critical notes.» American Journal of Agricultural Economics, 53:356-359. 


\section{Anexo}

\section{CUADRO A1}

Contrastes de raíces unitarias estacionales ${ }^{\mathrm{a}, \mathrm{b}}$

\begin{tabular}{lccc}
\hline & P $_{\text {Productor }}$ & P $_{\text {Consumo }}$ & Valor crítico (5\%) \\
\hline$\Pi_{1}$ & $-2,17$ & $-2,28$ & $-2,81$ \\
$\Pi_{2}$ & $-3,09$ & $-4,62$ & $-2,81$ \\
$\Pi_{3} \cap \pi_{12}$ & 8,73 & 13,66 & 4,44 \\
$\mathrm{~F}_{\mathrm{D} 1 \ldots \mathrm{D} 11}$ & 9,52 & 14,76 & 1,8 \\
Retardos & 3 & 5 & \\
$\mathrm{BG}(1)^{\mathrm{d}}$ & 0,54 & 3,02 & 3,84 \\
$\mathrm{BG}(12)^{\mathrm{d}}$ & 10,02 & 8,43 & \\
Normalidad $^{\mathrm{e}}$ & 2,85 & 3,36 & 3,84 \\
\hline
\end{tabular}

a El modelo incluye constante y ficticias estaciónales. Los valores críticos se han tomado de Franses y Hobijn (1997).

b $\pi_{1}$ es el estadístico para contrastar la existencia de raíz unitaria en la frecuencia regular; $\pi_{2}$ es el estadístico para contrastar la existencia de una raíz unitaria en la frecuencia estacional ${ }^{1} \frac{1}{2} ; \pi_{3} \cap \pi_{12}$ es el estadístico para contrastar la hipótesis conjunta de la existencia de raíces unitarias estacionales en todas las frecuencias.

c Estadístico F sobre la significatividad conjunta de las once variables ficticias. Este estadístico sólo sigue la distribución F habitual cuando se rechaza la existencia de raíces unitarias estacionales.

d Contraste de Autocorrelación Breusch-Godfrey (BG) sobre los residuos de la regresión, calculado para contrastar la presencia de autocorrelación de orden 1 y 12. Este estadístico se distribuye como una $\chi^{2}$ con 1 y 12 grados de libertad. e Contraste de normalidad de Jarque-Bera (JB). Este estadístico se distribuye como una $\chi^{2}$ con 1 grado de libertad. 